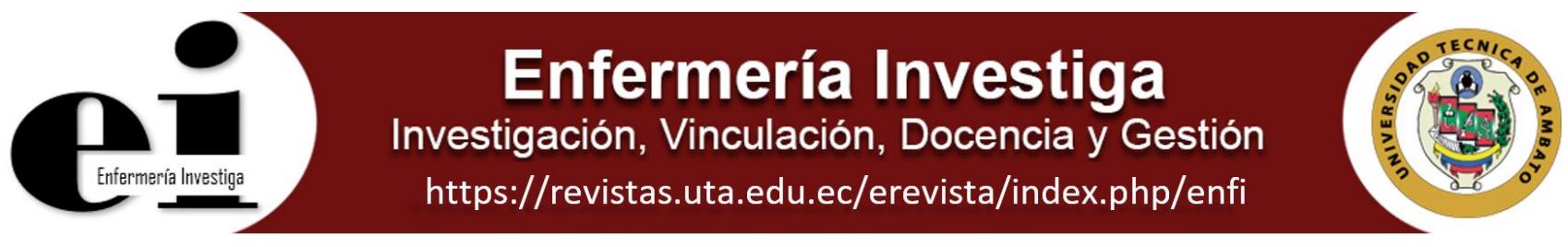

\title{
MARCADORES TUMORALES PRONÓSTICOS EN CÁNCER DE MAMA: BAX y Bcl-2
}

\section{PROGNOSTIC TUMOR MARKERS IN BREAST CANCER: BAX and Bcl-2}

Lizette Elena Leiva Suero ${ }^{1}$ https://orcid.org/0000-0001-9899-029X, Yenddy Carrero Castillo1 https://orcid.org/00000003-4050-4468, Graciela de las Mercedes Quishpe Jara² https://orcid.org/

0000-0002-0461-0602, Sandra Villacís Valencia ${ }^{1}$ https://orcid.org/0000-0002-9584-0680, Jesús Onorato Chicaiza Tayupanta ${ }^{1}$ https://orcid.org/0000-0002-5777-2971, Susana Arias Tapia ${ }^{3}$ https://orcid.org/0000-0002-5337-3156

1GRUPO DE INVESTIGACIÓN ACADÉMICA Y CIENTÍFICA EN CIENCIAS BIOMÉDICAS CON PROYECCIÓN SOCIAL KUSKIYKUY YACHAY SUNTUR. Proyecto de Investigación PFCS42 Caracterización de marcadores genéticos e inmunológicos con valor diagnóstico, pronóstico y terapéutico en cáncer de mama y cuello uterino. Carrera de Medicina. Facultad de Ciencias de la Salud. Universidad Técnica de Ambato-Ecuador.

${ }^{2}$ GRUPO DE INVESTIGACIÓN ACADÉMICA Y CIENTÍFICA EN CIENCIAS BIOMÉDICAS CON PROYECCIÓN SOCIAL KUSKIYKUY YACHAY SUNTUR. Proyecto de Investigación PFCS42 Caracterización de marcadores genéticos e inmunológicos con valor diagnóstico, pronóstico y terapéutico en cáncer de mama y cuello uterino. Carrera de Enfermería. Facultad de Ciencias de la Salud. Universidad Técnica de Ambato-Ecuador

${ }^{3}$ Proyecto de Investigación PFCS42 Caracterización de marcadores genéticos e inmunológicos con valor diagnóstico, pronóstico y terapéutico en cáncer de mama y cuello uterino. Carrera de Laboratorio Clínico. Facultad de Ciencias de la Salud. Universidad Técnica de Ambato-Ecuador

2477-9172 / 2550-6692 Derechos Reservados @ 2022 Universidad Técnica de Ambato, Carrera de Enfermería. Este es un artículo de acceso abierto distribuido bajo los términos de la Licencia Creative Commons, que permite uso ilimitado, distribución y reproducción en cualquier medio, siempre que la obra original es debidamente citada.

Recibido:15 de noviembre 2021

Aceptado: 27 de diciembre 202

\section{RESUMEN}

Introducción: El carcinoma de mama representa el 16\% de todos los cánceres femeninos, cada año en las Américas, más de 462,000 mujeres son diagnosticadas y casi 100,000 mueren por esta causa, por lo que la detección de nuevos marcadores pronósticos se hace imprescindible para el desarrollo de nuevas terapias oncológicas. Objetivo: Identificar los marcadores tumorales con fines pronósticos en cáncer de mama en células de cultivo de carcinoma de mama MCF7. Método: Se desarrolló un estudio explicativo longitudinal y prospectivo observacional para identificar marcadores tumorales con valor pronóstico en modelos celulares MCF7 de Cáncer de Mama, se utilizarán métodos estadísticos descriptivos e inferenciales. Resultados: Se identificó un predominio de $\mathrm{Bcl}-2$ en las células de cultivo MCF7 de Carcinoma de Mama. Conclusión: La identificación de BAX y Bcl-2 en modelos celulares MCF 7 de Carcinoma de Mama permite evaluar nuevas opciones terapéuticas relacionadas con la inducción de la apoptosis. La predominancia $\mathrm{Bcl}-2$ pudiera constituir un factor de mal pronóstico y menor supervivencia debido a su efecto inhibidor de la apoptosis, donde subyacen alteraciones mitocondriales, que inciden en la regulación del nivel de calcio intracelular, o por su efecto antioxidante.

Palabras clave: Neoplasias de la mama, Pronóstico,
Apoptosis, Proteína de Susceptibilidad a Apoptosis Celular

\section{ABSTRACT}

Introduction: Breast carcinoma represents $16 \%$ of all female cancers, each year in the Americas, more than 462,000 women are diagnosed and almost 100,000 die from this cause, so the detection of new prognostic markers is essential for the development of new oncological therapies. Objective: To identify tumor markers for prognostic purposes in breast cancer in MCF7 breast carcinoma culture cells. Method: A longitudinal and prospective observational explanatory study was developed to identify tumor markers with prognostic value in MCF7 cell models of Breast Cancer, descriptive and inferential statistical methods will be used. Results: A predominance of $\mathrm{Bcl}-2$ was identified in the MCF7 culture cells of Breast Carcinoma. Conclusion: The identification of BAX and Bcl-2 in MCF 7 cell models of Breast Carcinoma allows to evaluate new therapeutic options related to the induction of apoptosis. The Predominance bcl-2 could constitute a factor of poor prognosis and lower survival due to its inhibitory effect of apoptosis, where mitochondrial alterations underlie, which affect the regulation of the level of intracellular calcium, or for its antioxidant effect.

Keywords: Breast Neoplasms, Prognosis, Apoptosis, Cellular Apoptosis Susceptibility Protein 


\section{INTRODUCCIÓN}

El carcinoma de mama representa el $16 \%$ de todos los cánceres femeninos, con una incidencia variable, cuyas tasas normalizadas por edad alcanzan valores de 99,4 por 100000 habitantes con incremento sostenido en todas las regiones a nivel mundial. Cada año en las Américas, más de 462,000 mujeres son diagnosticadas con cáncer de mama, y casi 100,000 mueren a causa de esta enfermedad. La continuidad en las tendencias actuales, representará que para el año 2030, el número de mujeres diagnosticadas con cáncer de mama aumente en un $34 \%$ en las Américas. Se considera en América Latina y el Caribe, el cáncer más común entre las mujeres y es el segundo en mortalidad. El diagnóstico precoz y los avances terapéuticos han logrado una mayor supervivencia con cáncer de mama. Sin embargo, muchos países de América Latina y el Caribe continúan teniendo un acceso limitado a estas novedosas intervenciones. Las tasas de supervivencia del cáncer mamario varían mucho en todo el mundo, desde el $80 \%$ o más en América del Norte, Suecia y Japón, pasando por un $60 \%$ aproximadamente en los países de ingresos medios, hasta cifras inferiores al $40 \%$ en los países de ingresos bajos. Las bajas tasas de supervivencia observadas en los países poco desarrollados pueden explicarse principalmente por la falta de programas de detección precoz, que hace que un alto porcentaje de mujeres acudan al médico con la enfermedad ya muy avanzada, pero también por la falta de servicios adecuados de diagnóstico y tratamiento (1-5).

En el Ecuador, la incidencia de Cáncer de mama según estadísticas de Globocan 2018, hubo 28.058 casos nuevos de cáncer, en promedio existen 165 casos de cáncer en todas sus variedades por cada 100.000 mujeres. Los cánceres más comunes en mujeres: los cánceres más comunes según su incidencia son: mama $2787(18,2 \%)$, cuello uterino 1612 (10,6\%), tiroides $1374(9 \%)$, estómago $1225(8 \%)$ y colorrectal 1123 (7,4\%). Según datos del Instituto Nacional de Estadísticas y Censos (INEC), el cáncer de mama es una de las principales causas de muerte en las mujeres ocupando el lugar número 11 de la lista de causas generales de muerte femenina en el 2017. De las 3.430 defunciones reportadas por esta causa entre el 2012 y 2017 , el $99,3 \%$ de las personas que murieron por esta enfermedad fueron mujeres. Solo en el año 2017 se registraron 670 defunciones por cáncer de mama en mujeres y 3 en hombres, que corresponde a una tasa de mortalidad de 3,99 defunciones por cada 100.000 habitantes. Según los registros del INEC 2017, la mortalidad por localización de los tumores en orden descendente en mujeres fue: mama, estómago, cuello uterino, bronquios y pulmón, y de hígado y vías biliares (1-3).

Debido al comportamiento agresivo de algunas variedades y dado que la mama es un órgano accesible para el diagnóstico temprano, el cáncer de mama es objeto permanente de estudios en relación con nuevos métodos de diagnóstico y tratamiento

El estadio clínico (TNM): tamaño tumoral, estado de los ganglios linfáticos y metástasis a distancia, ha sido el factor pronóstico de sobrevida más eficaz. El estado de los ganglios linfáticos es el parámetro más importante que define la terapéutica a seguir. Algunas pacientes $(25-30 \%$ de los casos) sin metástasis ganglionar en el momento del diagnóstico, pero a los 10 años tienen una recurrencia de la enfermedad, lo que plantea una decisión dilemática desde el punto de vista terapéutico: administrar o no quimioterapia, tratamiento hormonal o radiante. De ahí se desprende, entre otras, la necesidad de contar con otros factores o marcadores adicionales que brinden información válida acerca del comportamiento biológico del tumor y ayuden a seleccionar la terapéutica adecuada, a brindar a la paciente una mejor calidad de vida, predecir su sobrevida y monitorear los tratamientos (4-7).

El desarrollo vertiginoso de la biología molecular, ha posibilitado el estudio de los diferentes aspectos del comportamiento biológico de los tumores de la mama, a partir de diferentes alteraciones bioquímicas, moleculares y genéticas en las células neoplásicas; lo que ha posibilitado la identificación de nuevos marcadores tumorales que, sumados a los marcadores tradicionales aún vigentes, facilitan la selección de las estrategias terapéuticas beneficiosas personalizadas y favorecen el pronóstico de manera más precisa, con énfasis en el tiempo de sobrevida libre de enfermedad, los riesgos de metástasis y las posibles recidivas(5-10).

La jerarquización de los marcadores tumorales (MT) predictivos y pronósticos del cáncer de mama, fue consensuada en 1999 en EE UU por el Colegio de Patólogos Americanos y avala la utilización de los MT clásicos incluyéndolos en la primera categoría están el tipo histológico, el índice mitótico (por conteo), los receptores hormonales y la biopsia del ganglio centinela. En la Categoría II se hallan la determinación del oncogén c-erbB 2, la expresión del p53, los marcadores de la síntesis de ADN y la invasión vascular peritumoral. La Categoría III está representada por la determinación de la ploidía del ADN, evaluación del angiogénesis intratumoral, receptor para EGF, expresión de Bcl2, pS2 y Catepsina D (11-14).

Se ha documentado posteriormente que la determinación del angiogénesis tumoral expresada como densidad de microvasos (número) por campo microscópico tendría que estar ubicada en la categoría II. Numerosos estudios avalan su correlación con la presencia de metástasis locales o a distancia no sólo en mama sino en otros tumores malignos, por lo que debe situarse a la angiogénesis junto a invasión tumoral.

En la oncología clínica, se considera que la determinación del Antígeno Carcinoembrionario (CEA) sérico o sus anticuerpos, aportan información sobre las recidivas en el cáncer de mama.

Entre los MT de uso rutinario en Anatomía Patológica para el cáncer de mama destacan los ubicados en categoría I: el Receptor 2 del EGF humano (c-erbb-2), la densidad de la vascularización y el Antígeno Nuclear de Proliferación (PCNA) o Ki 67 (mib 1).

Resultan imprescindibles: tamaño del tumor, tipo histológico, pleomorfismo celular y nuclear, índice mitótico, presencia de necrosis, invasión vascular, estado de los receptores hormonales y de los ganglios linfáticos axilares. No obstante, resultan insuficientes para predecir el curso de la enfermedad.

Los avances de la biología molecular han aportado nuevos marcadores que ya se han ido incorporando a la práctica clínica y que brindan una importante información acerca del comportamiento biológico del tumor y la posible respuesta a la radio o quimioterapia, o sea, orientan la terapéutica a implementarse. Por lo que el uso de un panel de marcadores tumorales, proporciona información más certera que la que puede suministrar un solo factor (14-19).

Los marcadores tumorales son indicadores bioquímicos de la presencia de un tumor. Incluyen antígenos de superficie celular, proteínas citoplasmáticas, enzimas y hormonas. En la práctica clínica el término se utiliza referido a moléculas que pueden ser detectadas en plasma, fluidos corporales, tumores sólidos, células tumorales circulantes, ganglios linfáticos y médula ósea. Muestran valor diagnóstico, pronóstico y para identificar el estadio de la neoplasia, detectar recidivas, metástasis, monitorear respuesta al tratamiento y permiten realizar una pesquisa en la población Se consideran MT específicos, a aquellos antígenos producidos por las células tumorales o asociados a ellas que las hacen antigénicamente distintas a las células normales. Cualquier proteína de la célula 
Los Marcadores Séricos pasan al torrente sanguíneo y alcanzan concentraciones suficientes, su detección puede ser utilizada para realizar estudios en la población, diagnóstico, monitoreo de la respuesta terapéutica, indicadores pronósticos - detección de recidivas. En cáncer de mama son relativamente pocos los MT que pueden ser medidos en sangre. De ellos, los más utilizados en la actualidad son la mucina CA 15-3 y el CEA (25-30).

Para identificación de los MT localizados en los tejidos neoplásicos, se utilizan técnicas de inmunohistoquímica (IHQ), de inmunofluorescencia y enzimoinmunoensayo (ELISA) que tienen por finalidad poder visualizar y cuantificar aquellos marcadores contra los cuales se dispone de anticuerpos monoclonales específicos. Estas técnicas permiten identificar antígenos intracelulares y de membrana en cortes de tejido o en material obtenido por punción. Siendo una de las técnicas más utilizadas, la detección de receptores hormonales por inmunohistoquímica (IHQ), para evaluar la susceptibilidad del carcinoma mamario a la terapéutica antiestrogénica (1-10).

Resumiendo, en los últimos años ha existido un crecimiento del espectro de marcadores en cáncer de mama, debido a los avances de la biología molecular que está estudiando en profundidad los eventos de la carcinogénesis, la progresión tumoral y los mecanismos de producción de las metástasis. Estos marcadores pueden clasificarse en base a sus características biológicas (1-3):

Marcadores de proliferación: están presentes en determinadas fases del ciclo celular.

Factores de crecimiento y hormonas: estimulan el crecimiento tumoral.

- Receptores: su sobreexpresión o su presencia alterada puede estar presente en algunos tipos de células tumorales.

-Receptores para Estrógenos (RcE) cuya presencia es indicadora para instaurar tratamiento hormonal

- Angiogénesis y actores del microambiente: favorecen la progresión de la neoplasia.

- Moléculas de adhesión y expresión de proteasas: permiten la invasión y la metástasis.

- Oncogenes y genes supresores: su amplificación o sobrexpresión se asocia con la desregulación del crecimiento y la apoptosis.

- Proteínas inducidas por estrógenos: como Proteína de Choque Térmico 27 (Hsp27).

- Mucinas: su detección en la circulación se utiliza como índice de enfermedad residual y posibles recidivas.

\section{OBJETIVO}

Identificar marcadores tumorales con fines pronósticos en cáncer de mama en células de cultivo de carcinoma de mama MCF7.

\section{METODOS}

Se desarrolló un estudio explicativo longitudinal y prospectivo observacional para identificar marcadores tumorales con valor pronóstico en Cáncer de Mama, se utilizarán métodos estadísticos descriptivos e inferenciales.

Los ensayos se realizaron por triplicado, el programa estadístico a emplear será GraphPad Prism. Versión 7. Los resultados se presentarán como promedio \pm 1 desviación estándar, comparando el porcentaje de células positivas en relación con las células totales y la intensidad para cada ensayo realizado. Para cada análisis comparativo se empleará ANOVA más post test de presentaran Bonferroni considerando valores significativos de $p \leq 0.005$.

Descongelación y Cultivo celular

Los modelos celulares MFC-7 provenientes de Cáncer de Mama fueron almacenadas en medio de congelación (FBS con $10 \%$ de DMSO) y en atmosfera de nitrógeno líquido a $-196^{\circ} \mathrm{C}$ se descongelarán mediante el método indirecto. Se empleará
tripsina/EDTA al $0,25 \%$ a $37^{\circ} \mathrm{C}$ como método de disgregación celular. Se realizarán pases y se incubarán a condiciones estándar el tiempo requerido para alcanzar una confluencia del $70-80 \%$ para cada los experimentos posteriores.

Ensayos inmunocitoquímicos

Se realizarán ensayos inmunocitoquímicos mediante pruebas de microscopia de fluorescencia, para ello se realizará un subcultivo de células MCF7 a una concentración de 5×105 células/pozo que se dejarán crecer durante 48 horas.

Para la tinción se fijarán las células con paraformaldehido (PFA) al $4 \%$ y luego se permeabilizará la membrana con $1 \mathrm{ml}$ de PBS 1X 20mM Glicina 0,5\% Tritón 100X. Finalmente se añadirán 500ul de cada reactivo, a dilución 1:1000 según las recomendaciones de temperatura y agitación descritas por el fabricante. Finalmente se añadirá el medio de montaje Aqueous Mounting (Ultra Cruz) con DAPI y se observará en un microscopio de fluorescencia Leica-DMi8 (Leica Microsystems, Alemania).

\section{Consideraciones éticas}

La ciencia en general, la investigación y experimentación en particular, no tienen valor absoluto, sino que se ponen al servicio de las personas y de la sociedad. Se reconoce la necesidad de experimentación e investigación, pero se sostiene que el progreso científico no es motivo suficiente para justificar cualquier tipo de experimento. El respeto a los derechos humanos y el bienestar de la persona sujeto de investigación deben prevalecer siempre sobre los intereses de la ciencia y de la sociedad. Los principios básicos de la bioética están sustentados en el respeto a la dignidad humana, es por ello que se tomarán en cuenta las pautas establecidas por la Asociación Médica Mundial (AMM) a través de la promulgación de la Declaración de Helsinki como una propuesta de principios éticos para investigación médica en seres humanos, así mismo el Comité de Bioética de la Universidad Técnica de Ambato y se cumplirán las leyes establecidas en la República de Ecuador. A pesar de trabajar con modelos celulares se obtuvo la aprobación de un comité de Bioética avalado por el MSP, se mantuvieron los criterios éticos y de confidencialidad.

\section{RESULTADOS}

La proteína BCL 2 asociada (BAX) es uno de los principales factores efectores de la apoptosis en cáncer de mama, sin embargo, al contrario de lo que ocurre con su proteína antagónica $\mathrm{Bcl}-2$ existen muy pocos estudios publicados sobre el papel que juega BAX en la carcinogénesis de esta neoplasia.

$\mathrm{Bcl}-2$ es una proteína reguladora de la muerte celular programada (apoptosis), influye en ella, bloqueándola. Al interrumpirse este mecanismo, uno de cuyos fines es el control de la masa celular, las células afectadas adquieren una ventaja proliferativa, que las puede dotar de un fenotipo neoplásico, presentando resistencia a la apoptosis. La translocación $\mathrm{t}\{14 ; 18\}$ produce niveles altos inadecuados de la oncoproteína producto del gen, también denominada bcl-2; ésta se acumula en los tejidos y es susceptible de ser cuantificada. Sin embargo, bcl-2 no es el único gen implicado en el complejo mecanismo de la apoptosis; dentro de la familia de genes reguladores de la muerte celular, existen otros, con papeles promotores y supresores de la apoptosis, que se mantienen en un delicado equilibrio.

Expresión Bcl2 en células MCF-7

Tincion por inmunohistoquímica: Anticuerpo primario Anti BCL2 (Santa Cruz Biotechnology, Inc) /Anticuerpo secundario anti-mouse m-IgGk BP marcado con PE (ficoeritrina) Magnificación 630X y lectura con filtros Texa red $(595-615 \mathrm{~nm})$ y DAPI (489-513nm). 
Figura 1 Expresión de la Proteína que ayuda a controlar la supervivencia o destrucción celular por apoptosis (Bcl2) en una línea celular que conserva características del epitelio mamario diferenciado (Células (MCF-7)
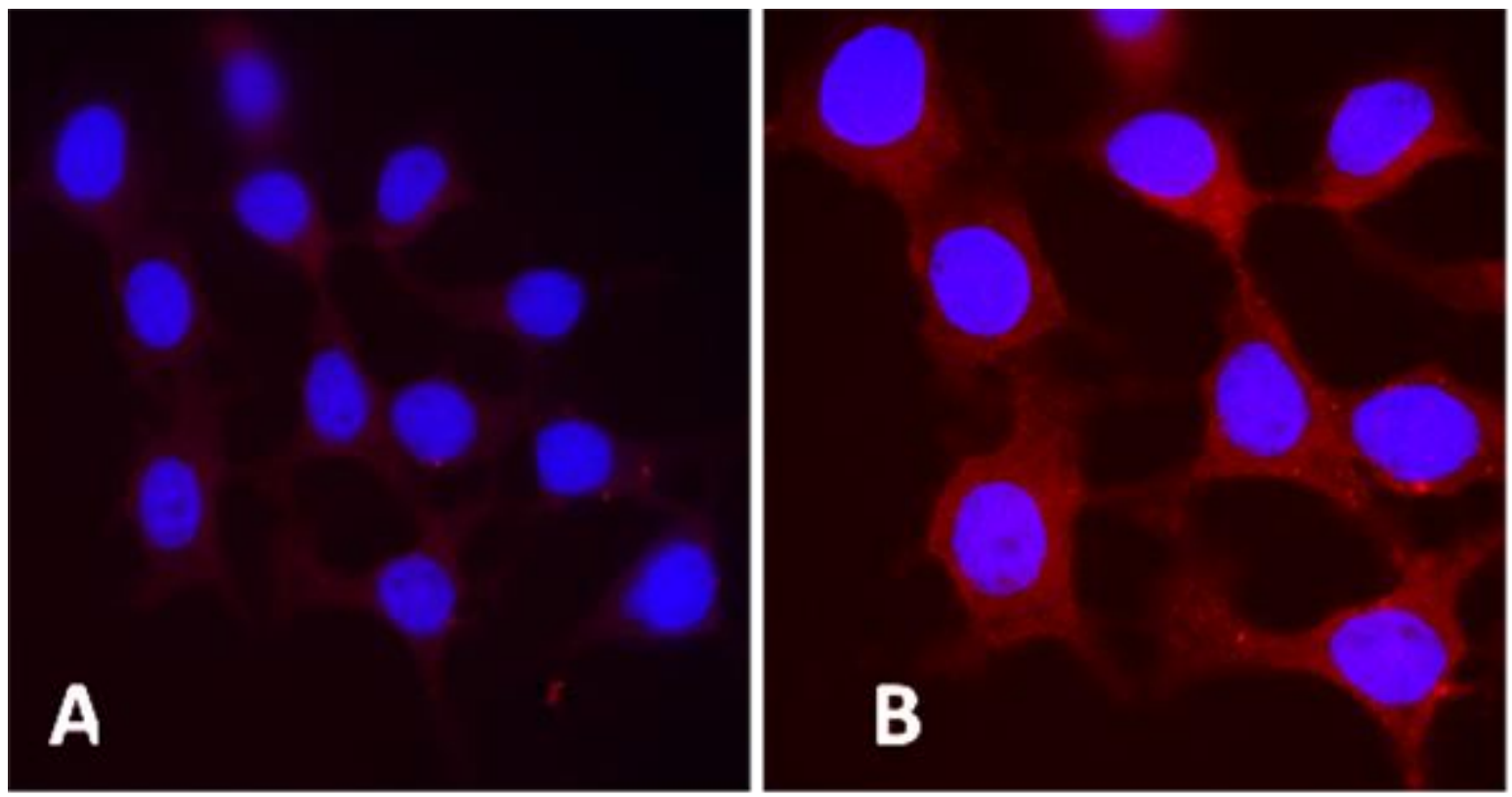

Fuente: Laboratorio de Investigaciones Facultad de Ciencias de la Salud de la Universidad Técnica de Ambato Expresión de BAX en células MCF-7

Tincion por inmunohistoquímica: Anticuerpo primario Anti BAX (Santa Cruz Biotechnology, Inc) /Anticuerpo secundario antimouse m-IgGk BP marcado con FITC Magnificación 630X y lectura con filtros GFP (475-509 nm) y DAPI (489-513nm)

Figura 2. Expresión de BAX Células MCF-7

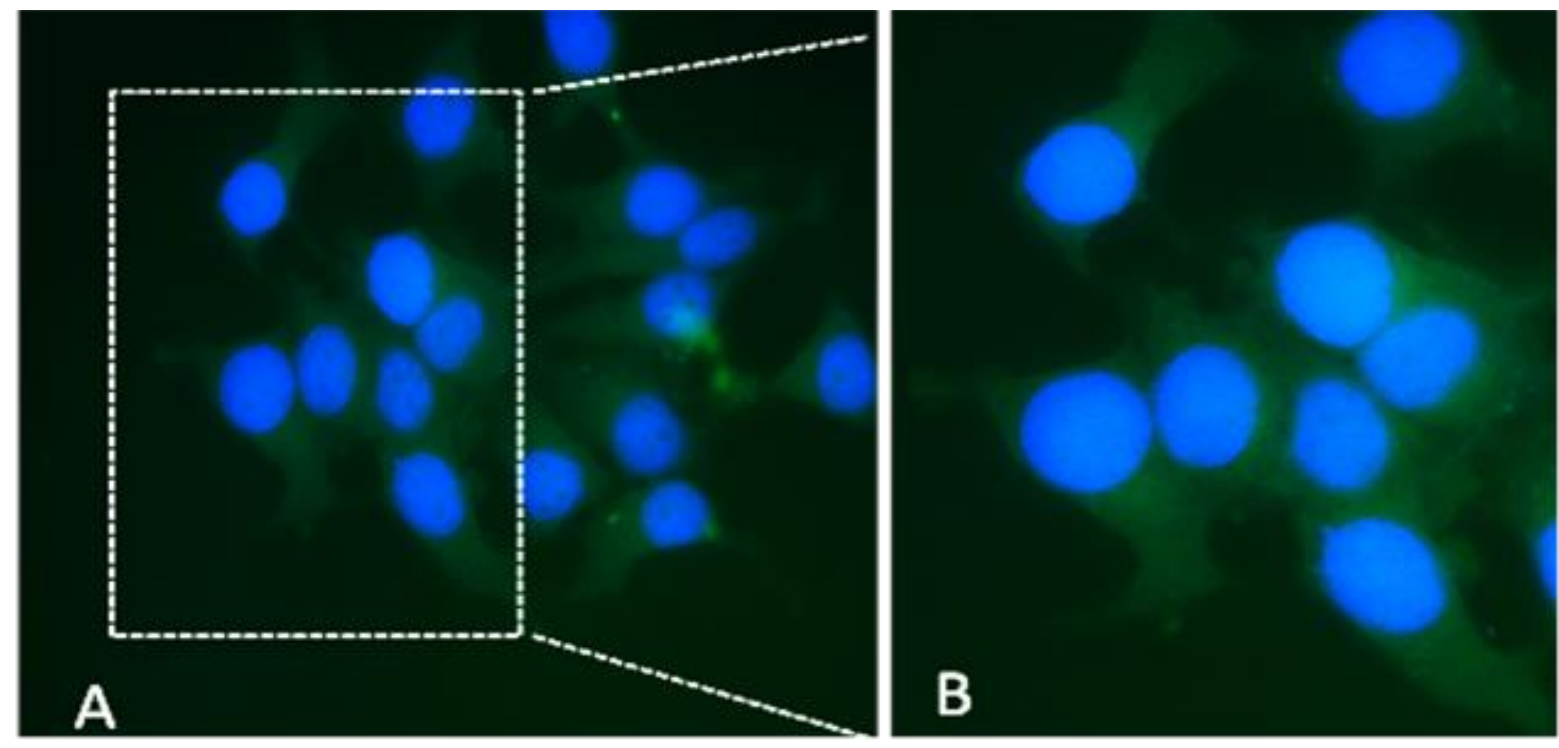

Fuente: Laboratorio de Investigaciones Facultad de Ciencias de la Salud de la Universidad Técnica de Ambato

Tabla 1. Valores obtenidos por técnicas de Inmunocitoquímica en células MCF 7 de Carcinoma de Mama provenientes de pacientes femeninas de la Zona 3

\begin{tabular}{|l|l|l|l|l|l|}
\hline \multirow{2}{*}{} & & & & & \\
Ct & & Target & Sample & Ct & Ct GAPDH \\
\hline 26,83 & BAX & Control & 26,83 & 26,520 \\
\hline 26,83 & BAX & Control & 26,83 & 26,520 \\
\hline 29 & Bcl-2 & Control & 29 & 26,520 \\
\hline
\end{tabular}




\begin{tabular}{|l|l|l|l|l|}
\hline 29 & Bcl-2 & Control & 29 & 26,520 \\
\hline
\end{tabular}

Fuente: Laboratorio de Investigaciones Facultad de Ciencias de la Salud de la Universidad Técnica de Ambato Gráfico 1 Expresión de BAX en Células MCF-7

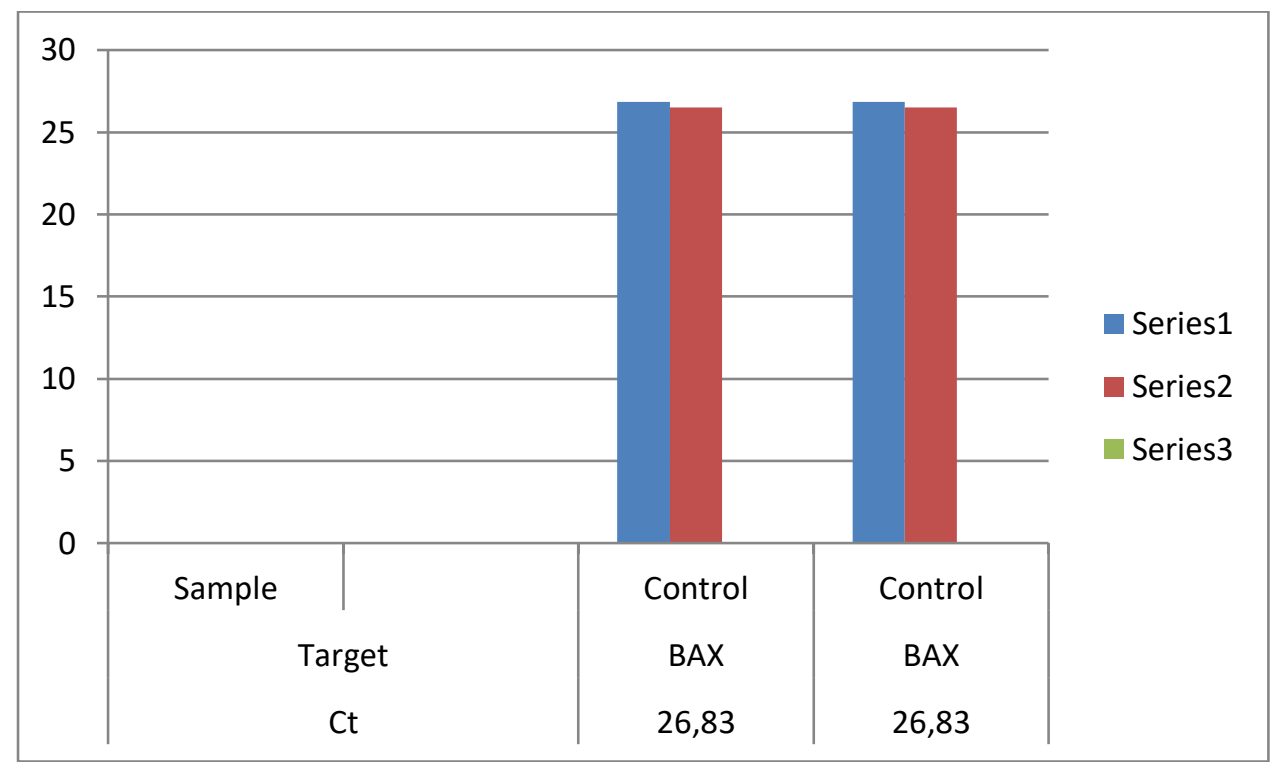

Fuente: Laboratorio de Investigaciones Facultad de Ciencias de la Salud de la Universidad Técnica de Ambato

Gráfico 2 Expresión de Bcl-2 en Células MCF-7

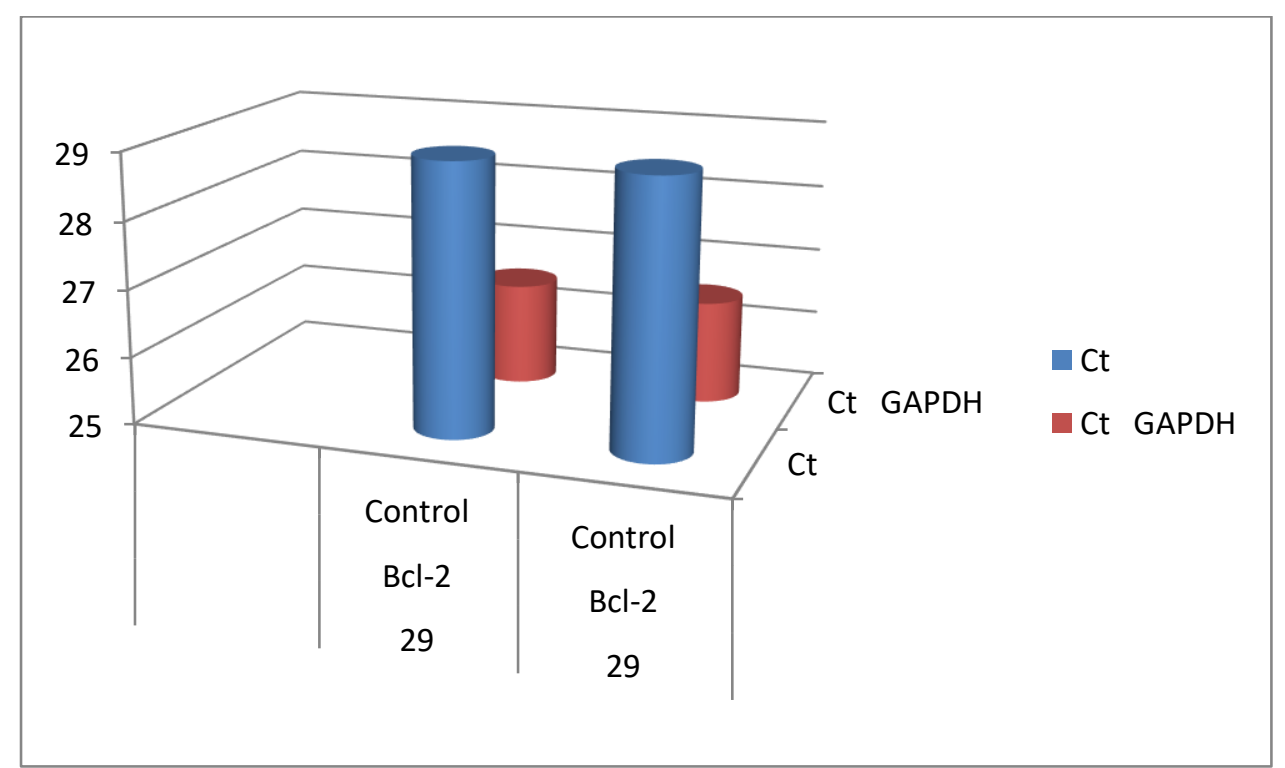

Fuente: Laboratorio de Investigaciones Facultad de Ciencias de la Salud de la Universidad Técnica de Ambato

Para un índice BAX/Bcl-2 de 0,92, lo cual representa un predominio de efecto antiapoptótico.

\section{DISCUSIÓN}

En la mama, al igual que en otros tejidos, Bcl-2 se relaciona con el desarrollo y la diferenciación epitelial, expresándose intensamente en la mama en desarrollo, de forma que en este estadio su presencia no implica un fenotipo tumoral (1-5).

La base molecular por la que Bcl-2 inhibe la apoptosis no está completamente dilucidada. Pero se sugiere la existencia de determinadas alteraciones mitocondriales, que inciden en su posible rol como reguladora del nivel de calcio intracelular, o también se ha relacionado con que en determinadas circunstancias ejerce acción antioxidante, bloqueando la muerte mediada por radiaciones gamma y/o determinadas hormonas, entre otros. Aunque la posible implicación de bcl-2 en la patogénesis del carcinoma de mama no ha sido aún comprobada, en otros tejidos se ha confirmado su aparición en lesiones pre-neoplásicas y preinvasivas. La adquisición de un fenotipo tumoral que originaría el carcinoma mamario, podría guardar relación con diferentes factores etiológicos: desregulación de la apoptosis por desbalance entre genes anti-apoptosis (Bcl-2) y genes pro- apoptosis (BAX). Las relaciones de $\mathrm{Bcl}-2$ con otros oncogenes, como cerbB-, c-myc, y p53, plantea la posibilidad de que Bcl-2 estuviera implicado en un mecanismo progresivo adquisición de mutaciones acumulativas por las células con positividad para $\mathrm{Bcl}-2$, que pudiera ir transformando el fenotipo celular hasta hacerlo tumoral. Se ha considerado que Bcl-2 confiere longevidad a las 
células, por tal motivo pudiera incrementar el tiempo de que disponen para sufrir alteraciones mutagénicas de cualquier tipo que conduzcan a la aparición de esta neoplasia (6-14). No obstante, el fenotipo tumoral en el que estaría implicado $\mathrm{Bcl}-2$ sería menos agresivo que los inducidos por otros mecanismos. Debido a una estrecha correlación entre la expresión de $\mathrm{Bcl}-2$ y la positividad para receptores de estrógenos y progesterona, con negatividad para la expresión de la proteína supresora de tumores (p53), asociado a una supervivencia más prolongada (15-22).

Por otro lado, se ha identificado la asociación entre la expresión de Bcl-2 y el alto índice de proliferación con Mib-1, considerado de mal pronóstico (15-18).

La negatividad para Bcl-2 observada en tumores receptor de estrógenos (RE) negativos con alta proliferación celular, caracterizaría un grupo de tumores que no requeriría Bcl-2 para inhibir la apoptosis y prolongar de esta forma la vida celular, respondiendo a otros factores de crecimiento independientes del control hormonal (15-26).

Se ha documentado además una relación inversa entre la oncoproteína Bcl-2 y la expresión del oncogén supresor p53, este último no sólo inhibe la proliferación celular, sino que también está involucrado en la inducción de la apoptosis en determinadas células tumorales (25-30).

En cuanto al significado pronóstico de $\mathrm{Bcl}-2$ no se mantiene como marcador independiente en el análisis multivariante en relación con los RE, p53, grado nuclear e histológico y Mib-1, pero sí se considera independiente del tamaño tumoral (1530).

Pudiera resultar de utilidad identificar la presencia de $\mathrm{Bcl}-2$ en la valoración de mujeres con CDI, con ganglios axilares negativos, para detectar grupos de pacientes con mal pronóstico, susceptibles de ser tratadas con ciclos de quimioterapia profiláctica, tras la cirugía. Hasta un $30 \%$ de enfermas con CDI, ganglios negativos y receptores hormonales positivos, presentan recidivas en los primeros 10 años. Estas enfermas pudieran beneficiarse de la administración de quimioterápicos, para lo cual es necesario identificar a este grupo; la estrecha relación de Bcl-2 con otros factores de buen pronóstico, podría ser utilizada, de forma que las enfermas de estas condiciones que tuviesen ausencia de $\mathrm{Bcl}-2$, serían las candidatas a la profilaxis, ya que como hemos visto, estos tumores tienden a la des diferenciación (14-22). Un campo de investigación abierto es el valor de la expresión de bcl-2 como factor predictivo de respuesta hormonal y quimioterápica, así como su relación e interacciones con estos agentes terapeúticos que frenan el estímulo hormonal y/o inducen apoptosis, como el tamoxifeno (18-27).

La mayor parte de los estudios que han valorado la expresión de Bax en carcinomas de mama han asociado la expresión aumentada de esta proteína con una peor diferenciación tumoral (25-28). Binder et al. asocian la expresión de Bax con características negativas histopatológicas especialmente cuando la expresión de $\mathrm{Bcl}-2$ está desregulada concomitantemente (26-30). Otros estudios que has valorado la expresión conjunta de ambas proteínas, encuentran, sin embargo, que es la pérdida de expresión de ambas proteínas lo que confiere un comportamiento más agresivo a los tumores (15-25). Otros autores, que también han estudiado el efecto conjunto de ambas proteínas, no encuentran significado pronóstico en su relación como tampoco en la expresión aislada de Bax en relación con la sobrevivencia (15-30).

Algunos autores que han investigado la expresión de ambas proteínas tanto en carcinomas intraductales $(\mathrm{Cl})$ como en carcinomas ductales infiltrantes (CDI), encuentran una clara expresión antagónica únicamente en $\mathrm{Cl}$, con un predominio de $\mathrm{Bcl}-2$ en tumores bien diferenciados y de Bax en carcinomas de alto grado. A medida que el tumor progresa se pierde la expresión de ambas proteínas a la vez que ésta se hace más coordinada. El balance entre Bcl-2 y Bax y su papel en el control de la apoptosis son factores importantes en la carcinogénesis y en la progresión tumoral, sin embargo, los mecanismos íntimos que controlan su interdependencia son poco conocidos (15-30).

La expresión aumentada del oncogén regulador de la muerte programada $\mathrm{Bcl}-2$ se observa más en tumores con marcadores biológicos favorables asociándose, con una supervivencia global y un periodo libre de enfermedad más largo, lo cual permite considerarle como un marcador favorable, aunque no independiente, en pacientes con ganglios linfáticos negativos (20-28).

Los casos con intensa positividad para BAX se correlacionan con tumores más agresivos y menor supervivencia.

Se ha identificado la asociación $\mathrm{Bcl}-2$ negativo/BAX intenso en el grupo de pacientes con los tumores más agresivos (15-30). En nuestro estudio predominó la presencia de $\mathrm{Bcl}-2$ con respecto al BAX lo cual sería considerado un factor de mejor pronóstico y mayor supervivencia.

\section{CONCLUSIÓN}

La identificación de BAX y Bcl-2 en modelos celulares MCF 7 de Carcinoma de Mama permite evaluar nuevas opciones terapéuticas relacionadas con la inducción de la apoptosis. La predominancia $\mathrm{Bcl}-2$ pudiera constituir un factor de mal pronóstico y menor supervivencia debido a su efecto inhibidor de la apoptosis, donde subyacen alteraciones mitocondriales, que inciden en la regulación del nivel de calcio intracelular, o por su efecto antioxidante. Estos hallazgos constituyen resultados preliminares los cuales permiten dilucidar la importancia de la identificación de los genes implicados en la ruta apoptótica y los procesos inflamatorios o neoplásicos persistentes en el microambiente celular, que predisponen a una acelerada progresión a la malignidad. Se espera a futuro estudiar muestras provenientes de pacientes para establecer las asociaciones etiológicas pertinentes en pro de desarrollar una inmunoterapia que potencie la activación de mecanismos propios de control genético en las células a partir de la inducción de la apoptosis a través de la identificación de genes tempranos y tardíos de la ruta apoptótica, con especial énfasis en BAX y Bcl-2, tomando además en consideración p53, Caspasas 3,7 y 8, así como la relación $\mathrm{BAX} / \mathrm{Bcl}-2$. De esto se deduce la importancia de identificar nuevos marcadores genéticos y moleculares que sean de utilidad pronóstica y que constituyan la base de nuevas opciones terapéuticas, así como para el seguimiento de las pacientes e identificación de resistencia a los tratamientos oncológicos convencionales.

\section{AGRADECIMIENTO}

GRUPO DE INVESTIGACIÓN ACADÉMICA Y CIENTÍFICA EN CIENCIAS BIOMÉDICAS CON PROYECCIÓN SOCIAL KUSKIYKUY YACHAY SUNTUR. Proyecto de Investigación PFCS42 Caracterización de marcadores genéticos e inmunológicos con valor diagnóstico, pronóstico y terapéutico en cáncer de mama y cuello uterino.

\section{REFERENCIAS.}

1. Carrasco, MC Pérez. Expresión de las proteínas bax y bcl2 en células de cáncer de mama in vitro tratadas con amaranthus hipochondriacus. repositorio.uta.edu.ec; 2020: http://repositorio.uta.edu.ec/handle/123456789/31536 
2. Cristina-Pérez, M, Acosta, JA, Carrero, Y. Expresión de las proteínas BAX y BCL2 en células de cáncer de mama in vitro tratadas. Investigación Clínica. researchgate.net; 2021; 62 (Supl 3): https://www.researchgate.net/profile/YenddyCarrero/publication/356412663_Expression_of_BAX_and_BCL2_proteins_in_vitro_breast_cancer_cells_treated_with_Amarant hus_hypochondriacus/links/61990d193068c54fa5085aa9/Expression-of-BAX-and-BCL2-proteins-in-vitro-breast-cancer-cellstreated-with-Amaranthus-hypochondriacus.pdf

3. Sánchez, R, Schneider, E, Martínez, G. Cáncer de mama Modalidades terapéuticas y marcadores tumorales. Cuadernos de Cirugía. revistas.uach.cl; 2018; 22 (1): http://revistas.uach.cl/index.php/cuadcir/article/view/2506

4. Carrasco-Brocal, C. Marcadores tumorales en el cáncer de mama. tauja.ujaen.es; 2017: $<$ http://tauja.ujaen.es/handle/10953.1/5486>

5. Ortega, MA Esparza. Determinación de marcadores tumorales por Inmunohistoquímica en muestras histopatológicas de pacientes con diagnóstico de Cáncer de Mama durante el periodo2014-2017 en el Hospital San Francisco de Quito. dspace.uce.edu.ec; 2019: http://www.dspace.uce.edu.ec/handle/25000/19627

6. Vargas, DA Morales. Valor predictivo de los marcadores tumorales Ca 15-3 y Antígeno Carcinoembrionario CEA en el tratamiento y seguimiento de las pacientes con cáncer de mama atendidas en el Hospital de Especialidades Eugenio Espejo durante el año 2016. dspace.uce.edu.ec; 2018: http://www.dspace.uce.edu.ec/handle/25000/17130

7. Romero, L Romero, Araujo, Y Salas. Marcadores tumorales en el cáncer de mama canino y su similitud con el cáncer de mama humano. 2017

8. Bonilla-Sepúlveda, ÓA. Marcadores tumorales en cáncer de mama. Revisión sistemática. Ginecología y Obstetricia de México. medigraphic.com; 2020; 88 (12): https://www.medigraphic.com/cgi-bin/new/resumen.cgi?IDARTICULO=97249

9. García, NR, Devora, GM. Marcadores tumorales en cáncer de mama: CA 15-3 y antígeno carcinoembrionario. Revista Mexicana de Mastología. medigraphic.com; 2016; 6 (1): https://www.medigraphic.com/cgibin/new/resumen.cgi?IDARTICULO=67324

10. Salvaa, AL. Cáncer de mama: utilidad pronóstica de los nuevos marcadores tumorales. sespm.es: http://www.sespm.es/wpcontent/uploads/revista/2003_16_2/4.pdf

11. Santesteban, P. Marcadores tumorales celulares como factores pronósticos en pacientes con cáncer de mama sin afectación axilar. sespm.es: http://www.sespm.es/wp-content/uploads/revista/1994_7_1/4.pdf

12. Zornoza, G. Valor de algunos marcadores tumorales (CEA, CA-15, MCA) en el pre y postoperatorio del cáncer de mama. sespm.es: https://www.sespm.es/wp-content/uploads/revista/1994_7_3/4.pdf

13. Gordon, JB. Marcadores tumorales en el cáncer de mama \{11. sespm.es:http://www.sespm.es/wpcontent/uploads/revista/1996_9_4/6.pdf

14. Peña-Blanco, A, García-Sáez, AJ. Bax, Bak and beyond-mitochondrial performance in apoptosis. The FEBS journal. Wiley Online Library; 2018:https://doi.org/10.1111/febs.14186

15. Große, L, Wurm, CA, Brüser, C, Neumann, D. Bax assembles into large ring-like structures remodeling the mitochondrial outer membrane in apoptosis. The EMBO Science. embopress.org; 2016; 35:402-413: https://doi.org/10.15252/embj.201592789 16. O'Neill, KL, Huang, K, Zhang, J, Chen, Y. Inactivation of prosurvival Bcl-2 proteins activates Bax/Bak through the outer mitochondrial membrane. Genes \& Development. genesdev.cshlp.org; 2016; 30:973-988: http://genesdev.cshlp.org/content/30/8/973.short

17. Huang, K, O'Neill, KL, Li, J, Zhou, W, Han, N, Pang, X. BH3-only proteins target BCL-xL/MCL-1, not BAX/BAK, to initiate apoptosis. Cell research. nature.com; 2019; 29: 942-952: https://www.nature.com/articles/s41422-019-0231-y

18. Garner, TP, Amgalan, D, Reyna, DE, Li, S. Small-molecule allosteric inhibitors of BAX. Nature chemical biology. nature.com; 2019; 15: 322-330: https://www.nature.com/articles/s41589-018-0223-0

19. Vince, JE, Nardo, D De, Gao, W, Vince, AJ, Hall, C. The mitochondrial apoptotic effectors BAX/BAK activate caspase-3 and7 to trigger NLRP3 inflammasome and caspase-8 driven IL-1 $\beta$ activation. Cell reports. Elsevier; 2018; 25(9): 2339-2353: https://www.sciencedirect.com/science/article/pii/S221112471831725X

20. Fernández, ÁF, Sebti, S, Wei, Y, Zou, Z, Shi, M. Disruption of the beclin 1-BCL2 autophagy regulatory complex promotes longevity in mice. Nature. nature.com; 2018;558:136-140: https://www.nature.com/articles/s41586-018-0162-7

21. Hata, AN, Engelman, JA, Faber, AC. The BCL2 family: key mediators of the apoptotic response to targeted anticancer therapeutics. Cancer discovery. AACR; 2015;5(5):475-87: https://cancerdiscovery.aacrjournals.org/content/5/5/475.short

22. Nechiporuk, T, Kurtz, SE, Nikolova, O, Liu, T, Jones, CL. The TP53 apoptotic network is a primary mediator of resistance to BCL2 inhibition in AML cells. Cancer discovery. AACR; 2019;9(7):910-25: https://cancerdiscovery.aacrjournals.org/content/9/7/910.abstract

23. Lok, SW, Whittle, JR, Vaillant, F, Teh, CE, Lo, LL. A phase lb dose-escalation and expansion study of the BCL2 inhibitor venetoclax combined with tamoxifen in ER and BCL2-positive metastatic breast cancer. Cancer discovery. AACR; 2019: 9 (3): https://cancerdiscovery.aacrjournals.org/content/9/3/354.abstract

24. Cui, J, Placzek, WJ. Post-transcriptional regulation of anti-apoptotic BCL2 family members. International journal of molecular sciences. mdpi.com; 2018;19 (1):308: https://www.mdpi.com/255874

25. Pekarsky, Y, Balatti, V, Croce, CM. BCL2 and miR-15/16: from gene discovery to treatment. Cell Death \& Differentiation. nature.com; 2018; 25:21-26: https://www.nature.com/articles/cdd2017159

26. Murakawa, T, Okamoto, K, Omiya, S, Taneike, M. A mammalian mitophagy receptor, Bcl2-L-13, recruits the ULK1 complex to induce mitophagy. Cell reports. Elsevier; 26 2019; https://www.sciencedirect.com/science/article/pii/S2211124718319806

27. Sánchez, EJ Millones. Rol de la expresión de BCL2 en la respuesta a tratamiento de cáncer de mama triple negativo Hospital Nacional Arzobispo $\quad$ Loayza $2018 . \quad$ repositorioacademico.usmp.edu.pe; https://repositorioacademico.usmp.edu.pe/handle/20.500.12727/6503

28. Nicolau, P, Corominas, JM, Macià, F, Carreras, R. P53 y otros factores predictores de la carga axilar en los distintos inmunofenotipos del cáncer de mama. Revista de Senología y Patología Mamaria. Elsevier; 202 ; 34 (2):70-76: https://www.sciencedirect.com/science/article/pii/S021415822030178X

29. Castillo, L, Bonilla, F, Reigosa, A, Fernández, Á. Expresión de p53 y Bcl-2 en carcinoma ductal infiltrante de mama localmente avanzado. Su relación con el subtipo intrínseco molecular como factor pronóstico. Investigación Clínica. redalyc.org; $2018 ; 59$ (4): 335-338: https://www.redalyc.org/jatsRepo/3729/372960204005/372960204005.pdf 
30. Bonilla-Sepulveda, OA. Breast papillary lesions: Clinical, pathological, and prognostic study in 144 cases in Medellin, Colombia/Lesoes papilares mamarias: estudo clinico-patologico e prognostico em 144 casos de Medellin, Colombia/Lesiones papilares de mama: estudio clinico-patologico y sobre pronostico en 144 casos de Medellín, Colombia. Medicina UPB. go.gale.com;

2021; 40

https://go.gale.com/ps/i.do?id=GALE\%7CA680550627\&sid=googleScholar\&v=2.1\&it=r\&linkaccess=abs\&issn=01204874\&p=IF $M E \& s w=w$ 